\title{
Physical activity trends and metabolic health outcomes in people living with HIV in the US, 2008-2015 ל⿱
}

\author{
Amanda L. Willig ${ }^{\mathrm{a}, *}$, Allison R. Webel ${ }^{\mathrm{b}}$, Andrew O. Westfall ${ }^{\mathrm{c}}$, Emily B. Levitan ${ }^{\mathrm{d}}$, Heidi M. Crane ${ }^{\mathrm{e}}$, \\ Thomas W. Buford $^{\mathrm{f}}$, Greer A. Burkholder ${ }^{\mathrm{a}}$, James H. Willig ${ }^{\mathrm{a}}$, Aaron J. Blashill ${ }^{\mathrm{g}}$, Richard D. Moore ${ }^{\mathrm{h}}$, \\ W. Christopher Mathews ${ }^{\mathrm{i}}$, Anne Zinski ${ }^{\mathrm{a}}$, Josh Muhammad ${ }^{\mathrm{j}}$, Elvin H. Geng ${ }^{\mathrm{k}}$, Sonia Napravnik ${ }^{1}$, Joseph J. Eron \\ $\mathrm{m}$,
} Benigno Rodriguez $^{\mathrm{n}}$, Marcas M. Bamman $^{\mathrm{o}}$, E. Turner Overton $^{\mathrm{a}}$

a Division of Infectious Diseases, University of Alabama at Birmingham, Birmingham, AL, United States of America

${ }^{\mathrm{b}}$ Frances Payne Bolton School of Nursing, Case Western Reserve University, Cleveland, OH, United States of America

${ }^{\mathrm{c}}$ Department of Biostatistics, University of Alabama at Birmingham, Birmingham, AL, United States of America

d Department of Epidemiology, University of Alabama at Birmingham, Birmingham, AL, United States of America

e Department of Medicine, Harborview Medical Center, University of Washington, Seattle, WA, United States of America

${ }^{\mathrm{f}}$ Division of Gerontology, Geriatrics, and Palliative Care, University of Alabama at Birmingham, Birmingham, AL, United States of America

${ }^{g}$ Department of Psychology, San Diego State University, San Diego, CA, United States of America

${ }^{\mathrm{h}}$ Johns Hopkins Bloomberg School of Public Health, Baltimore, MD, United States of America

${ }^{\mathrm{i}}$ Department of Medicine, University of California San Diego, San Diego, CA, United States of America

${ }^{\mathrm{j}}$ Department of Nutrition Sciences, University of Alabama at Birmingham, Birmingham, AL, United States of America

${ }^{\mathrm{k}}$ School of Medicine, University of California, San Francisco, CA, United States of America

${ }^{1}$ Department of Medicine, University of North Carolina, Chapel Hill, NC, United States of America

$\mathrm{m}$ Departments of Medicine and Epidemiology, University of North Carolina, Chapel Hill, NC, United States of America

${ }^{n}$ Department of Medicine, Case Western Reserve University, Cleveland, $\mathrm{OH}$, United States of America

o Department of Cell, Developmental, and Integrative Biology, University of Alabama at Birmingham, Birmingham, AL, United States of America

\section{A R T I C L E I N F O}

Article history:

9 February 2020

9 February 2020

Keywords:

Cardiovascular disease

Obesity

Diabetes

Multimorbidity

Health outcomes

Health disparities

\begin{abstract}
A B S T R A C T
Despite its potential to improve metabolic health outcomes, longitudinal physical activity (PA) patterns and their association with cardiometabolic disease among people living with HIV (PLWH) have not been well characterized. We investigated this relationship among PLWH in the Centers for AIDS Research Network of Integrated Clinical Systems with at least one PA self-report between 2008 and 2015. The 4-item Lipid Research Clinics PA instrument was used to categorize habitual PA levels as: Very Low, Low, Moderate, or High. We analyzed demographic differences in PA patterns. Multivariable generalized estimating equation regression models were fit to assess longitudinal associations of PA with blood pressure, lipid, and glucose levels. Logistic regression modeling was used to assess the odds of being diagnosed with obesity, cardiovascular disease (CVD), cerebrovascular disease, hypertension, diabetes, or multimorbidity. A total of 40,462 unique PA assessments were provided by 11,719 participants. Only $13 \%$ of PLWH reported High PA, while $68 \%$ reported Very Low/Low PA at baseline and did not increase PA levels during the study period. Compared to those reporting High PA, participants with Very Low PA had almost 2-fold increased risk for CVD. Very Low PA was also associated with several risk factors associated with CVD, most notably elevated triglycerides (odds ratio 25.4), obesity (odds ratio 1.9), hypertension (odds ratio 1.4), and diabetes (odds ratio 2.3; all $p<0.01$ ). Low levels of PA over time among PLWH are associated with increased cardiometabolic disease risk.
\end{abstract}

\footnotetext{
Abbreviations and acronyms: ANOVA, analysis of variance; BF, black female; BM, black male; BMI, body mass index; CVD, cardiovascular disease; CFAR, Centers for AIDS Research;

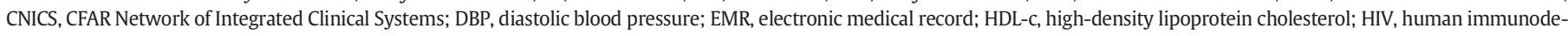

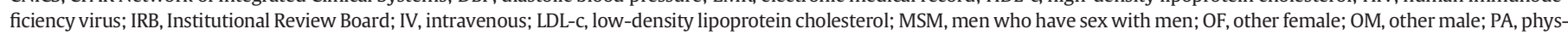
ical activity; PLWH, people living with HIV; RNA, ribonucleic acid; SBP, systolic blood pressure; WF, white female; WM, white male.

th Statement of conflict of interest: see page 176 .

* Address reprint requests to Amanda L. Willig PhD, RD, BBRB 203, 845 19th Street South, University of Alabama at Birmingham, Birmingham, AL $35294-2050$.

E-mail address: awillig@uabmc.edu (A.L. Willig).
} 


\section{Contents}

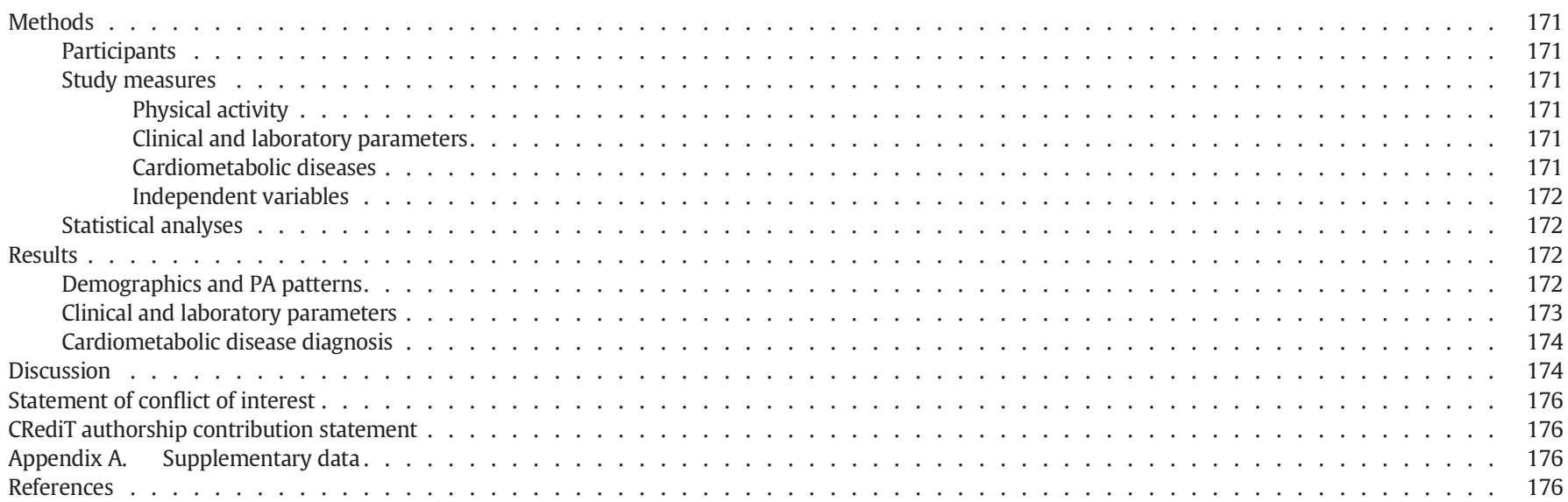

An increasing burden of non-AIDS cardiometabolic diseases is welldocumented among people living with HIV $(\mathrm{PLWH}) .{ }^{1-3}$ We reported that while most PLWH gain weight during the first six months of antiretroviral therapy use, many continue to gain weight for up to two years after beginning treatment. ${ }^{4}$ In the current United States epidemic, over $2 / 3$ of PLWH are diagnosed as overweight/obese (25\% obese), $40 \%$ with hypertension, and $6 \%$ cardiovascular disease (CVD) ${ }^{4-6}$ CVD and other cardiometabolic diseases are associated with increased mortality risk in adults living with and without HIV infection ${ }^{7,8}$; thus these alarming results highlight a need for effective interventions to prevent and treat cardiometabolic disease among PLWH.

One potential intervention is to increase habitual physical activity (PA), which is associated with decreased mortality and improved health outcomes among the general population. ${ }^{9,10}$ Unfortunately, PLWH have lower physical fitness levels compared to other vulnerable populations, ${ }^{11}$ and longitudinal studies conducted in PLWH have primarily focused on specific exercise interventions rather than habitual physical activity. ${ }^{12,13}$ Thus it is unknown if the amount of PA needed for meaningful improvement in cardiometabolic disease biomarkers and outcomes is different for PLWH versus those without HIV. Additionally, long-term data regarding PA habits of PLWH are lacking, while cross-sectional studies of PA levels that include smaller sample sizes from single sites reveal a wide disparity in self-reported activity levels. ${ }^{14-16}$ To develop effective PA strategies, we require a greater understanding of the longitudinal PA patterns reported by PLWH, and the minimal amount of activity needed to reduce cardiometabolic disease risk. Therefore, the objectives of this study were to (1) describe PA patterns among PLWH, and (2) determine which cardiometabolic disease biomarkers/diagnoses are most influenced by PA patterns in this population. To accomplish these objectives, we analyzed PA and cardiometabolic disease data collected over 7.5 years from 11,719 PLWH in the multisite Centers for AIDS Research (CFAR) Network of Integrated Clinical Systems (CNICS) cohort of PLWH in the United States.

\section{Methods}

This retrospective analysis was conducted in the United States from CNICS. ${ }^{17}$ CNICS is a nationally distributed clinical cohort that includes over 32,000 PLWH receiving routine clinical care. At regular intervals, CNICS sites provide comprehensive data on demographics, laboratory values, pharmaceutical history, HIV/AIDS clinical events, and comorbid conditions collected from electronic medical records and other data sources, including the CNICS clinical assessment of standardized Patient-Reported Outcomes measured at 4-6 month intervals ${ }^{18,19}$ at seven CNICS sites. A rigorous, systematic quality assurance process is in place to maintain this centralized database.

\section{Participants}

This study includes all CNICS participants who completed a clinical assessment between January 2008 (first PA measures available) and mid-July 2015. Inclusion criteria were: (1) at least one PA instrument completed, (2) $\geq 19$ years of age, and (3) height/weight were available. Written, informed consent for CNICS was obtained from all study participants and documented at each site, and the protocol was approved by the Institutional Review Board (IRB) at each site. Ethical approval for this study was provided by the IRB at the University of Alabama at Birmingham. Procedures were followed in accordance with the ethical standards of the responsible committee on human experimentation (institutional and national) and with the Helsinki Declaration of 1975, as revised in 2013.

\section{Study measures}

\section{Physical activity}

Participants completed the 4-question, validated Lipid Research Clinics (LRC) instrument to provide an estimate of self-reported PA. ${ }^{20}$ The LRC asks about respondents' perception, compared to peers, of PA amount/intensity at work and outside of work. A 4-point scoring system is used to classify activity levels as (1) high PA (vigorous activity $\geq 3$ times weekly), (2) moderate PA (active, but vigorous activity $<3$ times weekly), (3) low PA (no vigorous activity but light activity perceived as equivalent to peers), or (4) very low PA (sedentary). The 4-point scoring previously identified physiologically relevant group differences in $\mathrm{VO}_{2 \max }$ : High PA $41.9 \mathrm{ml} / \mathrm{kg}^{-1} / \mathrm{min}^{-1}$, Moderate $39.1 \mathrm{ml} / \mathrm{kg}^{-1} / \mathrm{min}^{-1}$, Low $33.2 \mathrm{ml} /$ $\mathrm{kg}^{-1} / \mathrm{min}^{-1}$, and Very Low $32.9 \mathrm{ml} / \mathrm{kg}^{-1} / \mathrm{min}^{-1}$. $^{20}$

\section{Clinical and laboratory parameters}

Vital signs and laboratory values closest to the date ( \pm 90 days) of each PA assessment were included. Systolic (SBP) and diastolic (DBP) blood pressure were measured at the beginning of patient care encounters as part of routine clinical care. As participant lab draws occurred throughout the day, both fasting and non-fasting glucose and lipid values (total cholesterol, LDL-c, HDL-c, triglycerides) were included.

\section{Cardiometabolic diseases}

We focused on five cardiometabolic outcomes with a prevalence of at least $2 \%$ in the CNICS cohort: obesity, CVD, cerebrovascular disease, 
hypertension, and diabetes - as well as multimorbidity. [1] Obesity. Participants were considered obese with a body mass index (weight $[\mathrm{kg}] /$ height $\left.\left[\mathrm{m}^{2}\right]\right) \geq 30$. [2] CVD. Participants were classified as having CVD if they were diagnosed with a myocardial infarction centrally adjudicated within CNICS. ${ }^{21}$ Briefly, two physicians who are experienced myocardial infarction reviewers adjudicate each event based on available EMR data. If there is a discrepancy in the outcome, a third reviewer will also adjudicate the event. Adjudication protocols were not yet developed for other CVD conditions, thus recorded diagnoses (yes/no) in the EMR of chronic ischemic heart disease and congestive heart failure were considered for CVD. [3] Cerebrovascular disease. Similar to other large HIV cohorts, adjudicated stroke data were not available in CNICS. Thus, a participant was considered to have cerebrovascular diseases with a diagnosis in the EMR. [4] Hypertension. Hypertension was based on the CNICS standard definition of a diagnosis of hypertension and the presence of any antihypertensive medication, or the average of at least 2 systolic blood pressure measurements $\geq 140 \mathrm{~mm} \mathrm{Hg}$ or diastolic blood pressure measurements $\geq 90 \mathrm{~mm} \mathrm{Hg}$ over 12 months. As we were unable to confirm whether all patients with hypertension or CVD were taking medications as prescribed, we included participants with both pharmaceutically treated and untreated conditions. [5] Diabetes. We defined diabetes based on the following criteria: a) hemoglobin A1c $\geq 6.5$ OR b) use of a diabetes-specific medication such as insulin OR c) use of a diabetes-related medication frequently but not exclusively used to treat diabetes (e.g. biguanides) in the setting of also having a diabetes diagnosis. ${ }^{22}$

Multimorbidity. Multimorbidity was computed as the presence of two or more of the above 5 cardiometabolic diseases as well as chronic kidney disease and cancer (HIV and non-HIV related). Chronic kidney disease was added to multimorbidity criteria despite $<2 \%$ population prevalence due to its association with physical activity and risk factors of poorly controlled HTN and obesity. ${ }^{23}$ Presence of chronic kidney disease was defined as an estimated glomerular filtration rate $<60 \mathrm{ml} / \mathrm{min} / 1.73 \mathrm{~m} 2$ for $>3$ months ( 2 values $>90$ days apart without an intervening normal value), or a charted diagnosis of Stage 2, 3, 4, or 5 kidney disease. The low number of total cancer cases in the CNICS cohort prohibited us from analyzing the association of physical activity with cancer risk. However, cancer diagnoses (classified as dichotomous yes/no) were added to the multimorbidity criteria since physical activity may be associated with cancer risk and metabolic risk factors contributing to development of other cardiometabolic conditions may also increase cancer risk. $^{24,25}$

Biomarkers. We further investigated the association of physical activity with available biomarkers of CVD risk, hypertension, and diabetes: Total Cholesterol, LDL-cholesterol, HDL-cholesterol, triglycerides, systolic blood pressure, diastolic blood pressure, and glucose levels.

\section{Independent variables}

We examined patient demographic and HIV-related laboratory information including age, sex, self-reported race/ethnicity, CD4+ T-cell count, viral load, HIV transmission risk factor (intravenous drug use, men having sex with men [MSM]), antiretroviral therapy (ART) use (yes/no), insurance status (public, private, uninsured), and mortality (yes/no with confirmed date of death). The "d" drugs didanosine (DDI), stavudine (D4T), and zalcitabine (DDC) may contribute to mitochondrial toxicity and peripheral neuropathy that could impair PA ability ${ }^{26,27}$; thus we included a dichotomous variable (yes/no) representing whether a participant had ever been exposed to d-drugs. Since pharmaceutical therapy may impact the laboratory values evaluated, we documented each prescribed medicine that could affect results, with the list of medicines included for each laboratory value adjudicated by two clinical providers (GB and JW).

\section{Statistical analyses}

Descriptive characteristics were analyzed using chi-square tests, one-way analysis of variance (ANOVA) or Kruskal-Wallis ANOVA. Time of first PA assessment was considered the baseline for each participant. We computed prevalence, expressed as unadjusted percentages, for each cardiometabolic condition overall and by PA group. Because race/sex interactions were observed for cardiometabolic disease outcomes, a 6-level race/sex variable was created (black men, black women, white men, white women, other men, other women) for subsequent analyses. The "other" racial category was approximately $40 \%$ selfreported Asian/Pacific Islander, 40\% unidentified, 11\% American Indian, and $9 \%$ multiracial.

For each continuous laboratory outcome, generalized estimating equations were fit to estimate the longitudinal effect of PA on laboratory variables over time after controlling for covariates described under "independent variables". We also controlled for outcome-specific non-ART medications - antihyperlipidemic agents, antihypertensives, oral hypoglycemic agents, insulin - prescribed. For cardiometabolic disease outcomes, multivariable logistic regression was used to compute odds ratio and $95 \%$ confidence intervals $(\mathrm{CI})$ of the odds of developing each cardiometabolic condition controlling for the above covariates. Obesity was also a covariate in all models excluding those with multimorbidity and obesity as the dependent variable. For all analyses, site was included as a stratification factor and "High PA" was considered the referent group. With a multilevel modeling approach, adjustment for multiple comparisons was not performed. ${ }^{28}$ Due to the association of the ART medication zidovudine (azidothymindine) with myopathy we also conducted a sensitivity analysis including "ever used AZT" as a covariate; however, this variable was not associated with PA levels and had no impact on the association between PA and cardiometabolic outcomes. In all models, we further controlled for the following factors described under "independent variables": age, self-reported ethnicity, CD4+ Tcell count, viral load, HIV transmission risk factor, ART use, insurance status, and mortality. All data were analyzed using SAS version 9.4 (SAS Institute Inc., Cary, NC, USA) with a significance level of $p<0.05$.

\section{Results}

\section{Demographics and PA patterns}

Over $71 \frac{1}{2}$ years, a total of 40,462 unique PA assessments were provided by 11,719 CNICS participants, with 8157 providing $\geq 2$ reports, and 3029 completing $\geq 5$ reports. There were no significant differences in demographic characteristics by those who had completed different numbers of PA assessments; thus descriptive statistics for all 11,719 participants at their initial assessment are presented in Table 1. At time of first PA report, 26\% reported being Very Low active, 42\% Low active, $19 \%$ Moderate active, and $13 \%$ High active. Very Low and Low PA participants were younger, less likely to be male, more likely to report black race, and weighed more compared to other PA categories $(p<0.01)$. Approximately $54 \%$ of participants consistently reported Very Low/Low PA and 19\% consistently reported High PA at each reporting period; thus the majority (73\%) of respondents did not change PA levels during the observation period. There was no increase in Moderate/High PA over time. There was no difference in PA category frequency by year of first PA report, and no difference by PA category in the percentage of participants prescribed ART $(87-88 \%, p=0.50)$. Of note, individuals identifying as transgender were more likely to report Very Low PA $(p=0.04)$.

A slightly higher percentage of Very Low PA participants (23.7\%) had been exposed to d-drugs compared to other groups (range 20.5-21.4\%). Baseline CD4+ T-cell counts were significantly increased across PA categories $(p<0.01)$. Greater mortality was also observed in the Very Low PA group compared to all other groups. 
Table 1

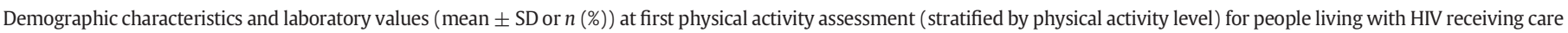
between January/2008 and July/2015.

\begin{tabular}{|c|c|c|c|c|c|c|}
\hline Variable & $\begin{array}{l}\text { Total } \\
n=11,719\end{array}$ & $\begin{array}{l}\text { Very Low } \\
n=3058\end{array}$ & $\begin{array}{l}\text { Low } \\
n=4957\end{array}$ & $\begin{array}{l}\text { Moderate } \\
n=2177\end{array}$ & $\begin{array}{l}\text { High } \\
n=1527\end{array}$ & $P$ value ${ }^{a}$ \\
\hline \multicolumn{7}{|l|}{ Demographics } \\
\hline Age (yrs) & $43.8 \pm 10.7$ & $44.9 \pm 10.2$ & $43.7 \pm 10.9$ & $42.8 \pm 10.5$ & $43.1 \pm 10.8$ & $<0.01$ \\
\hline \multicolumn{7}{|l|}{ Birth sex } \\
\hline Male & $9772(83.4)$ & 2409 (24.7) & 4067 (41.6) & $1956(20.0)$ & 1340 (13.7) & $<0.01$ \\
\hline Female & 1947 (16.6) & $649(33.3)$ & $890(45.7)$ & $221(11.4)$ & $187(9.6)$ & \\
\hline Transgender $^{\mathrm{b}}$ & & & & & & 0.04 \\
\hline Yes & $96(1.3)$ & $37(38.5)$ & $32(33.3)$ & $18(18.8)$ & $9(9.4)$ & \\
\hline No & 7104 (98.7) & $1842(25.9)$ & $2863(40.3)$ & $1445(20.4)$ & $954(13.4)$ & \\
\hline Race & & & & & & $<0.01$ \\
\hline \% Black & 3917 (33.4) & $991(25.3)$ & $1873(47.8)$ & $586(15.0)$ & 467 (11.9) & \\
\hline$\%$ White & $6735(57.5)$ & $1855(27.5)$ & $2643(39.2)$ & $1338(19.9)$ & $899(13.4)$ & \\
\hline$\%$ Other & 1067 (9.1) & $212(19.9)$ & $441(41.3)$ & $253(23.7)$ & $161(15.1)$ & \\
\hline \% Hispanic ethnicity & 1597 (13.7) & $358(11.8)$ & $666(13.5)$ & $350(16.2)$ & $223(14.8)$ & $<0.01$ \\
\hline Body mass index & $26.5 \pm 5.5$ & $27.0 \pm 6.3$ & $26.5 \pm 5.5$ & $26.3 \pm 4.8$ & $26.0 \pm 4.7$ & $<0.01$ \\
\hline Health insurance & & & & & & $<0.01$ \\
\hline Uninsured & $1167(9.9)$ & $279(23.9)$ & $477(40.9)$ & $240(20.6)$ & $171(14.6)$ & \\
\hline Public & $6171(52.7)$ & $1932(31.3)$ & $2552(41.3)$ & 1054 (17.1) & $633(10.3)$ & \\
\hline Private & $3083(26.3)$ & $455(14.8)$ & $1416(45.9)$ & $643(20.9)$ & $569(18.4)$ & \\
\hline Unknown & $1298(11.1)$ & $392(30.2)$ & $512(39.5)$ & $240(18.5)$ & $154(11.8)$ & \\
\hline Transmission risk factor & & & & & & $<0.01$ \\
\hline IVDU & $1771(15.1)$ & $579(32.7)$ & $697(39.4)$ & $324(18.3)$ & $171(9.6)$ & \\
\hline MSM & $6976(59.5)$ & $1632(23.4)$ & $2956(42.4)$ & 1393 (19.9) & $995(14.3)$ & \\
\hline Heterosexual & $2615(22.3)$ & $725(27.7)$ & $1170(44.7)$ & $402(15.4)$ & $318(12.2)$ & \\
\hline Other/unknown & $357(3.1)$ & $122(34.2)$ & $134(37.5)$ & $58(16.3)$ & $43(12.0)$ & \\
\hline Study site ${ }^{c}$ & & & & & & $<0.01$ \\
\hline UCSD & 3345 (28.6) & $908(27.1)$ & $1351(40.4)$ & $677(20.3)$ & $409(12.2)$ & \\
\hline$U A B$ & $2827(24.1)$ & $728(25.8)$ & $1358(48.0)$ & $402(14.2)$ & $339(12.0)$ & \\
\hline$U W$ & $1697(14.5)$ & $455(26.8)$ & $704(41.5)$ & $312(18.4)$ & $226(13.3)$ & \\
\hline$U C S F$ & 1093 (9.3) & $353(32.3)$ & $432(39.5)$ & $197(18.0)$ & $111(10.2)$ & \\
\hline Fenway Health & $1028(8.8)$ & $194(18.9)$ & $359(34.9)$ & $285(27.7)$ & $190(18.5)$ & \\
\hline UNC & $918(7.8)$ & $216(23.5)$ & $383(41.7)$ & $193(21.0)$ & $126(13.8)$ & \\
\hline John Hopkins & $811(6.9)$ & $204(25.2)$ & $370(45.6)$ & $111(13.7)$ & $126(15.5)$ & \\
\hline \multicolumn{7}{|l|}{ Clinical and laboratory parameters } \\
\hline $\mathrm{SBP}(\mathrm{mm} \mathrm{Hg})$ & $125.9 \pm 15.4$ & $125.2 \pm 15.9$ & $126.0 \pm 15.6$ & $126.4 \pm 14.9$ & $126.3 \pm 14.1$ & $<0.01$ \\
\hline DBP (mm Hg) & $78.7 \pm 10.3$ & $78.9 \pm 10.5$ & $78.9 \pm 10.3$ & $78.6 \pm 10.2$ & $78.2 \pm 9.7$ & 0.16 \\
\hline Total Cholesterol (mg/dL) & $177.2 \pm 42.7$ & $176.4 \pm 44.3$ & $177.8 \pm 43.3$ & $177.5 \pm 41.4$ & $176.1 \pm 39.2$ & 0.60 \\
\hline LDL-c $(\mathrm{mg} / \mathrm{dL})$ & $101.9 \pm 33.7$ & $100.6 \pm 34.6$ & $102.6 \pm 33.4$ & $102.4 \pm 33.8$ & $101.7 \pm 32.6$ & 0.20 \\
\hline HDL-c (mg/dL) & $43.8 \pm 16.4$ & $41.7 \pm 15.9$ & $44.3 \pm 16.2$ & $44.3 \pm 16.2$ & $46.1 \pm 17.7$ & $<0.01$ \\
\hline Triglycerides (mg/dL) & $176.5 \pm 180.5$ & $196.2 \pm 174.0$ & $176.3 \pm 211.6$ & $164.7 \pm 131.2$ & $154.0 \pm 130.8$ & $<0.01$ \\
\hline Glucose $(\mathrm{mg} / \mathrm{ml})$ & $95.1 \pm 30.9$ & $97.8 \pm 37.2$ & $94.7 \pm 30.2$ & $94.2 \pm 27.5$ & $92.2 \pm 22.9$ & $<0.01$ \\
\hline CD4+ T-cell count (cells/ $\mu \mathrm{l})$ & $506.7 \pm 294.3$ & $488.6 \pm 315.7$ & $508.7 \pm 293.3$ & $517.0 \pm 275.0$ & $521.9 \pm 278.2$ & $<0.01$ \\
\hline Plasma HIV RNA $<200$ copies $/ \mathrm{ml}$ & $7675(70.5)$ & $1928(68.5)$ & $3242(70.1)$ & $1472(72.6)$ & $1033(72.5)$ & $<0.01$ \\
\hline d-Drug exposure (ever) & & & & & & 0.01 \\
\hline Yes & $2533(21.6)$ & $724(28.6)$ & $1031(40.7)$ & $465(18.4)$ & $313(12.3)$ & \\
\hline No & $9186(78.4)$ & $2334(25.4)$ & $3926(42.7)$ & $1712(18.7)$ & $1214(13.2)$ & \\
\hline Death during observation period & & & & & & $<0.01$ \\
\hline Yes & $510(4.4)$ & $216(42.4)$ & $204(40.0)$ & $55(10.8)$ & $35(6.8)$ & \\
\hline No & $11,209(95.6)$ & $2842(25.4)$ & $4753(42.4)$ & $2122(18.9)$ & $1492(13.3)$ & \\
\hline
\end{tabular}

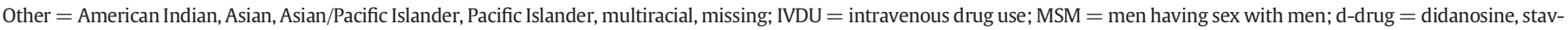
udine, zalcitabine.

SBP = systolic blood pressure; DBP = diastolic blood pressure; LDL-c = low-density lipoprotein cholesterol; HDL-c = high-density lipoprotein cholesterol.

a One-way ANOVA for continuous measures and chi-square for categorical measures.

b $n(\%)$ only for sites (UCSD, UAB, Fenway Health) collecting data on transgender identify up to $2015(n=7200)$.

c Study sites: UCSD = University of California, San Diego; UAB = University of Alabama at Birmingham; UW = University of Washington, Seattle; UCSF = University of San Francisco; UNC $=$ University of North Carolina at Chapel Hill.

\section{Clinical and laboratory parameters}

Laboratory values at time of first PA report are shown in Table 1. No significant differences were identified by PA group for DBP, total cholesterol, or LDL-c. Very Low PA participants had slightly lower SBP (125.2 $\mathrm{mm} \mathrm{Hg}$ ) compared to other groups (range 126.0-126.4 mm Hg). Those in the Very Low PA group presented with significantly lower HDL-c levels, higher triglycerides, and higher glucose compared to all other groups (all at $p<0.01$ ).

These differences persisted in longitudinal adjusted analyses (Fig. 1; Table 2). When compared to the High PA category, a significant stepwise decrease in HDL-c was observed with Moderate (Estimate $-0.71 ; 95 \% \mathrm{CI}-1.25,-0.17 ; p=0.009$ ), Low (Estimate $-1.03 ; 95 \%$ $\mathrm{CI}-1.54,-0.51 ; p<0.001$ ) and Very Low PA (Estimate $-1.56 ; 95 \% \mathrm{CI}$ $-2.17,-0.96 ; p<0.001)$. An increase in triglyceride levels was also observed for each PA group: Moderate (Estimate 7.78; 95\% CI 1.96, 13.59; $p=0.009$ ), Low (Estimate 16.12; 95\% CI 9.90, 22.34; $p<0.001$ ), and Very Low (Estimate 25.43; 95\% CI 15.64, 35.22; $p<0.001$ ). When glucose was analyzed, the Low PA (Estimate 1.35; 95\% CI 0.26, 2.45; $p=0.02$ ) and Very Low PA (Estimate $1.98 ; 95 \%$ CI 0.47, 3.49; $p=$ 0.01 ) groups had higher levels over time compared to the High PA group. Full models results are shown in Supplementary Table 1. 


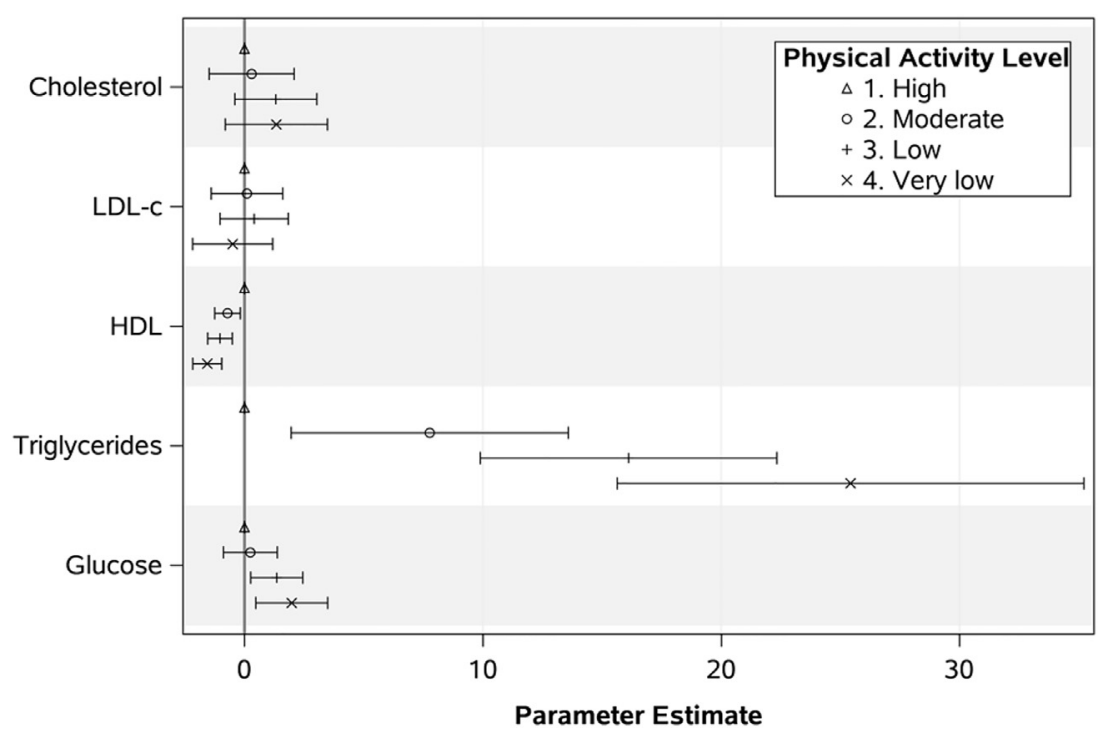

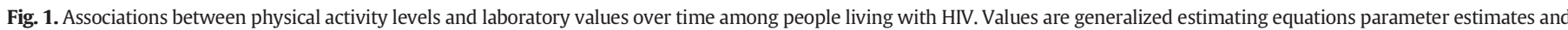

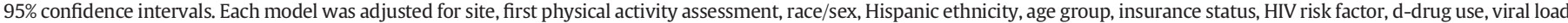
group, CD4 group, and lab specific non-antiretroviral therapy medications prescribed.

\section{Cardiometabolic disease diagnosis}

We observed significant differences in the prevalence of five cardiometabolic diseases and multimorbidity by PA group (Fig. 2). Over $1 / 3$ (35\%) of all participants were obese, but obesity prevalence was significantly different between groups: High PA (27\%); Moderate (31\%), Low (35\%,) and Very Low (41\%). Similar differences were observed with hypertension, CVD, and cerebrovascular diagnoses (Fig. 2). Finally, while $24 \%$ of the High PA group had cardiometabolic multimorbidity, greater prevalence was observed in other PA groups: Moderate $(27 \%, p=$ $0.003)$, Low ( $31 \%, p<0.001)$ and Very Low $(40 \%, p<0.001)$.

In adjusted models using High PA as the reference group (Table 2; Supplementary Table 2), only the Very Low PA group differed in prevalence of cerebrovascular disease (OR 1.76; 95\% CI 1.13-2.74). Both Low and Very Low PA were associated with greater risk for hypertension,
CVD, and diabetes, while a stepwise increase in risk of obesity and multimorbidity was observed across all PA categories. Participants with Very Low PA experienced 1.5-2 times greater risk of obesity, hypertension, CVD, and multimorbidity. In particular, the Very Low PA group had a 2.3 times greater risk of being diagnosed with diabetes.

\section{Discussion}

This investigation revealed that low PA levels are associated with increased risk for CVD and other related chronic diseases (i.e., obesity, hypertension, cerebrovascular disease, diabetes, and multimorbidity). Despite nation-wide efforts to promote PA, the majority (68\%) of PLWH reported Very Low/Low PA at baseline (compared to $49.8 \%$ of the general population), and did not increase PA during the study period. ${ }^{29}$ Unfortunately, only $13 \%$ of PLWH reported High PA, versus

Table 2

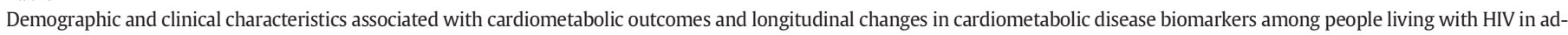

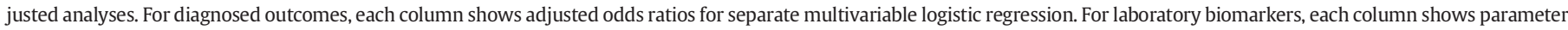
estimates and 95\% confidence limits for separate generalized estimating equations. All models also adjusted for site as a stratification factor.

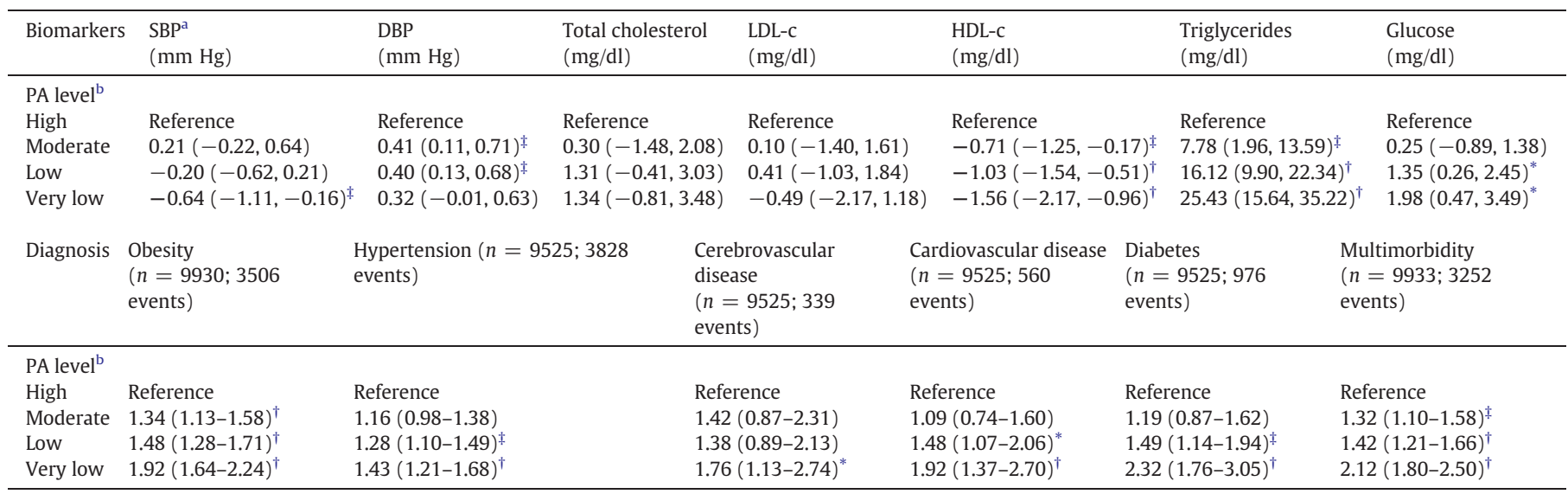

$* p<0.05$

$* p<0.01$.

$\dagger p<0.001$.

a $\mathrm{SBP}=$ systolic blood pressure; DBP = diastolic blood pressure; LDL-c = low-density lipoprotein cholesterol; HDL-c = high-density lipoprotein cholesterol; Multimorbidity is defined

as two or more diagnoses of obesity, CVD, cerebrovascular disease, hypertension, diabetes, chronic kidney disease, or cancer.

b $\mathrm{PA}=$ physical activity. 


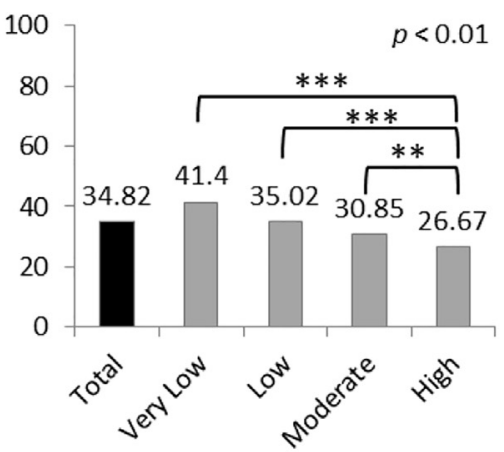

a. Obese $(\mathrm{BMI} \geq 30)$

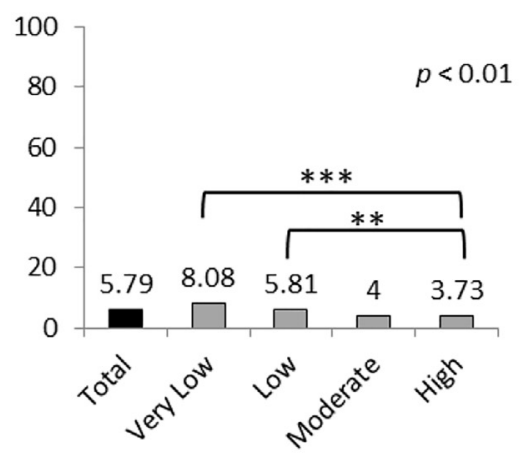

d. Cardiovascular Disease

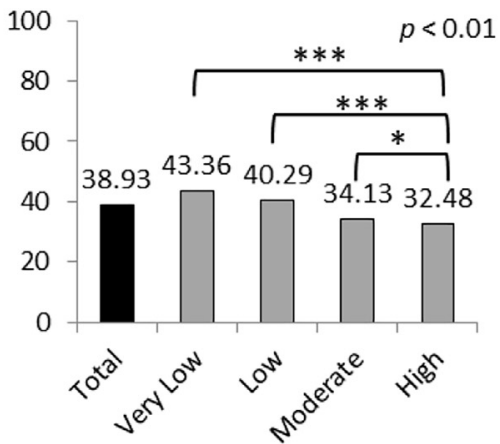

b. Hypertension

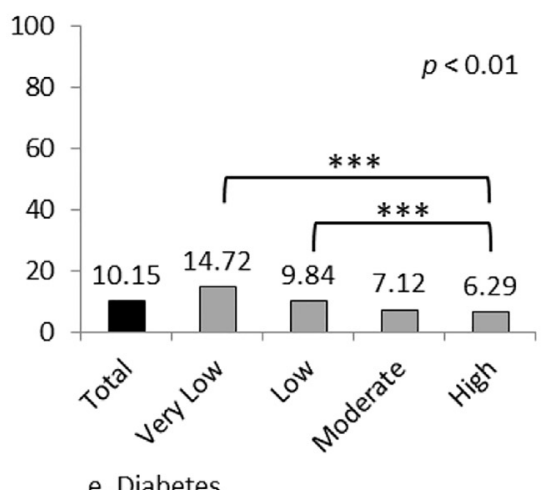

e. Diabetes

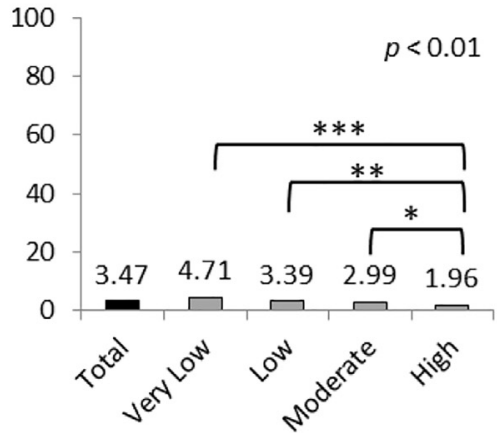

c. Cerebrovascular Disease

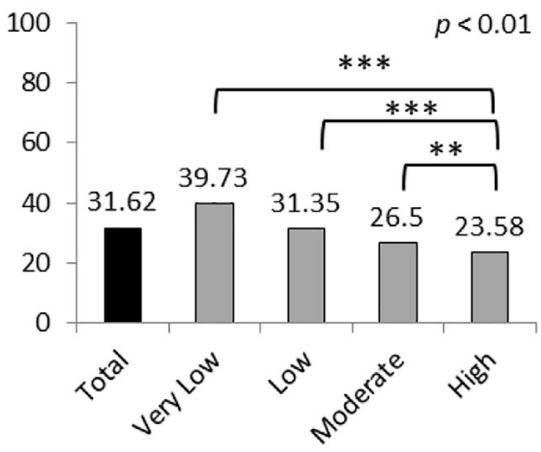

f. Multimorbidity

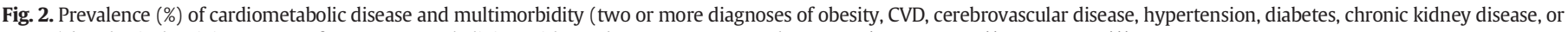
cancer) by physical activity category for 11,719 people living with HIV between $1 / 2008$ and $12 / 2015 .^{*}=p<0.05 ;^{* *}=p<0.01 ;{ }^{* * *}=p<0.001$.

$31.2 \%$ of the general population. ${ }^{29}$ Additionally, we found that participants in study sites in the Northeast (Fenway Health and Johns Hopkins) reported slightly higher levels of High PA. Overall, these results highlight the need to develop effective PA promotion strategies for PLWH.

We identified a consistent association of Very Low/Low PA with both cardiometabolic disease biomarkers and outcomes. The only differences in cardiometabolic disease risk for Moderate versus High PA were for obesity and multimorbidity, suggesting that even moderate PA may be sufficient to reduce risk for hypertension, cerebrovascular disease, CVD, and diabetes. In particular, reporting Very Low PA (versus High PA) was associated with higher glucose levels and almost 2.5 times increased diabetes risk.

A recent meta-analysis in HIV-negative adults observed reductions in type 2 diabetes risk of 26\% with Moderate PA and 53\% with High PA. ${ }^{30}$ Yarasheski et al. also reported that exercise augments the effects of pharmaceutical treatment in PLWH with diabetes. ${ }^{31}$ However, due to HIV-related chronic inflammation, pharmaceutical and lifestyle interventions may be less effective in PLWH versus HIV-negative groups. ${ }^{14,32}$ Monroe and colleagues ${ }^{14}$ reported that while low PA was associated with greater insulin resistance (e.g., higher HOMA-IR) regardless of HIV status, men with HIV still had higher HOMA-IR levels at equivalent levels of PA. Collectively, these results highlight uncertainty in exactly how much PA is required to achieve an equivalent benefit in diabetes prevention/treatment for this population.

PA level was associated with both hypertension and cerebrovascular disease. This association may have been attenuated by pharmaceutical interventions for blood pressure control. However, the tendency we observed to see lower blood pressure levels in those reporting Very Low PA could be confounded by a greater prevalence of uncontrolled viremia in this group, which was associated with lower odds of hypertension in our study (Supplementary Table 2) and others. ${ }^{33}$ The impact of PA frequency or type on HTN risk among PLWH who have not achieved virologic suppression remains to be fully investigated.
Our analysis also associated increased risk of CVD events and atherogenic lipid profile with Low/Very Low PA. An inverse association between PA levels and CVD risk factors/outcomes has been reported in PLWH and HIV-negative populations. ${ }^{34,35} \mathrm{PA}$ is also associated with higher HDL-c and lower triglycerides in HIV-negative individuals, possibly due to augmentation of lipoprotein lipase activity in skeletal muscle and adipose tissue. However, consistent with our results, two metaanalyses in HIV-negative individuals reported minimal declines in LDL-c and total cholesterol levels of 2900 men and 1715 women. ${ }^{36,37}$ Future investigations are required to determine to what extent PA can reduce the risk of CVD, and the amount and domains of PA required to meaningfully impact PLWH who have a higher-than-average risk for CVD.

Participants reporting Very Low PA tended to weigh more, have greater mortality, more exposure to d-drugs, and lower CD4+ T-cell counts than other groups. We previously reported that women with HIV have elevated body fat levels regardless of body weight, thus increasing PA may be particularly beneficial for $\mathrm{PLWH}^{38}$ Regarding the association with CD4+ T-cell count, small transient declines in CD4+ counts are observed with both periods of rest and strenuous activity in PLWH and -negative groups, possibly due to increased autophagy with hypoxia. ${ }^{39,40}$ However PA interventions are associated with increased CD4+ count in PLWH in some, but not all, studies. ${ }^{41,42}$ This study supports the potential of exercise to complement ART-related immunologic reconstitution as an area that requires additional exploration.

With regard to sex, race, and ethnicity, females had lower PA levels compared to males, and black participants more frequently reported Very Low/Low PA compared to other groups. Previous reports have identified similar patterns of sex or racial health disparities in PA levels in the general population and $\mathrm{PLWH},{ }^{16,43}$ confirming the need for programs to reduce sex and race disparities in PA. Interestingly, we also detected differences in PA for PLWH who identify as transgender among the 7200 participants reporting on transgender status. Within groups, 
only $9 \%$ of transgender participants identified as High PA compared to $13 \%$ of other participants, and $38 \%$ of transgender participants reported Very Low PA compared to $26 \%$ of others. Few investigations have explored unique aspects of health behaviors and risks among transgender individuals, particularly those with HIV. Fredriksen-Goldsen et al. ${ }^{44}$ reported lower PA levels in transgender adults over age 50 compared to lesbian, gay, and bisexual older adults, while Bryant and colleagues found that compared to other members of the LGBT community, transgender women in particular were at risk for low PA and poor diet. ${ }^{45}$ The milieu of hormonal and body composition changes, combined with unique barriers to healthy dietary and PA habits, could exacerbate cardiometabolic disease risk in this population. Unfortunately, our small sample limits the general applicability of these findings for transgender $\mathrm{PLWH}$. Additional research is needed to identify barriers, facilitators, and metabolic factors that contribute to disparities in PA for the transgender community.

Our findings are subject to certain limitations. Self-reported PA is less accurate than objectively measured PA and can underestimate Very Low/Low PA while overestimating High PA. ${ }^{46}$ However, this would possibly mean that Very Low/Low PA is present in more than the $68 \%$ of PLWH identified here, and further serves to emphasize the need for PA promotion in this population. Additionally, as our PA data collection only began in 2008 , and were unable to determine a reliable date of diagnosis for all cardiometabolic diseases, we are unable to consider the impact of PA habits across the life course and throughout HIV infection, which is an area of significant interest in disease prevention.

Despite these limitations, the present study benefited from several strengths, including a large, diverse sample size inclusive of several geographic regions across the United States, and repeated PA measures assessed over $7 \frac{1}{2}$ years of observation. The study included both biomarkers and outcomes of cardiometabolic disease and provides strong evidence that PA can have a meaningful impact on cardiometabolic disease in PLWH. These findings inform clinical practice by highlighting subgroups of PLWH that may benefit from additional PA promotion as well as risk factors that are more likely to be impacted when PA levels change. Additional scientific exploration that determines the appropriate domains and dose-response of PA for specific cardiometabolic disease, as well as strategies to most effectively implement PA programs among PLWH, holds great potential to improve outcomes and reduce health disparities in cardiometabolic disease risk among individuals living and aging with HIV.

\section{Statement of conflict of interest}

This work was supported by the National Institute of Nursing Research \#R01 NR018391, the National Institute of Allergy and Infectious Diseases \#P30-AI27767, the CNICS Research Network \#R24-AI067039, and the Mary Fisher CARE Fund. A.L.W. received support from the UAB Center for Outcomes and Effectiveness Research and Education \#TL1TR001418, UAB Nutrition Obesity Research Center [National Institute of Diabetes and Digestive and Kidney Diseases \#P30DK056336], and the UAB Center for Exercise Medicine. The views expressed are those of the authors, and funding agencies played no role in the design or interpretation of the research.

\section{CRediT authorship contribution statement}

Amanda L. Willig: Conceptualization, Writing - original draft, Visualization, Supervision, Project administration. Allison R. Webel: Conceptualization, Writing - original draft. Andrew 0. Westfall: Software, Formal analysis, Visualization, Writing - review \& editing. Emily B. Levitan: Formal analysis, Writing - review \& editing. Heidi M. Crane: Investigation, Resources, Writing - review \& editing. Thomas W. Buford: Writing - review \& editing. Greer A. Burkholder: Resources, Writing review \& editing. James H. Willig: Resources, Writing - review \& editing. Aaron J. Blashill: Writing - review \& editing. Richard D.
Moore: Resources, Writing - review \& editing. W. Christopher Mathews: Resources, Writing - review \& editing. Anne Zinski: Resources, Writing - review \& editing. Josh Muhammad: Writing - review \& editing. Elvin H. Geng: Resources, Writing - review \& editing. Sonia Napravnik: Resources, Writing - review \& editing. Joseph J. Eron: Writing - review \& editing. Benigno Rodriguez: Resources, Writing - review \& editing. Marcas M. Bamman: Writing - review \& editing. E. Turner Overton: Conceptualization, Writing - original draft.

\section{Appendix A. Supplementary data}

Supplementary data to this article can be found online at https://doi. org/10.1016/j.pcad.2020.02.005.

\section{References}

1. Effros RB, Fletcher CV, Gebo K, et al. Aging and infectious diseases: workshop on HIV infection and aging: what is known and future research directions. Clin Infect Dis 2008;47:542-553.

2. Palella Jr FJ, Baker RK, Moorman AC, et al. Mortality in the highly active antiretroviral therapy era: changing causes of death and disease in the HIV outpatient study. J Acquir Immune Defic Syndr 2006;43:27-34.

3. Vance DE, Mugavero M, Willig J, Raper JL, Saag MS. Aging with HIV: a crosssectional study of comorbidity prevalence and clinical characteristics across decades of life. The Journal of the Association of Nurses in AIDS Care: JANAC 2011;22:17-25.

4. Tate T, Willig AL, Willig JH, et al. HIV infection and obesity: where did all the wasting go? Antivir Ther 2012;17:1281-1289.

5. Willig AL, Westfall AO, Overton ET, et al. Obesity is associated with race/sex disparities in diabetes and hypertension prevalence, but not cardiovascular disease, among HIV-infected adults. AIDS Res Hum Retroviruses 2015;31:898-904.

6. Hernandez-Romieu AC, Garg S, Rosenberg ES, Thompson-Paul AM, Skarbinski J. Is diabetes prevalence higher among HIV-infected individuals compared with the general population? Evidence from MMP and NHANES 2009-2010. BMJ Open Diabetes Res Care 2017:5, e000304.

7. Zhang D, Tang X, Shen P, et al. Multimorbidity of cardiometabolic diseases: prevalence and risk for mortality from one million Chinese adults in a longitudinal cohort study. BMJ Open 2019;e024476:9.

8. Feinstein MJ, Bahiru E, Achenbach C, et al. Patterns of cardiovascular mortality for HIV-infected adults in the United States: 1999 to 2013. Am J Cardiol 2016;117: 214-220.

9. Arem H, Moore SC, Patel A, et al. Leisure time physical activity and mortality: a detailed pooled analysis of the dose-response relationship. JAMA Intern Med 2015;175:959-967.

10. Lear SA, Hu W, Rangarajan S, et al. The effect of physical activity on mortality and cardiovascular disease in 130000 people from 17 high-income, middle-income, and low-income countries: the PURE study. Lancet 2017;390:2643-2654.

11. Vancampfort D, Mugisha J, Rosenbaum S, et al. Cardiorespiratory fitness levels and moderators in people with HIV: a systematic review and meta-analysis. Prev Med 2016;93:106-114

12. Cade WT, Reeds DN, Overton ET, et al. Pilot study of pioglitazone and exercise training effects on basal myocardial substrate metabolism and left ventricular function in HIV-positive individuals with metabolic complications. HIV Clin Trials 2013;14:303312.

13. Garcia A, Fraga GA, Vieira Jr RC, et al. Effects of combined exercise training on immunological, physical and biochemical parameters in individuals with HIV/AIDS. J Sports Sci 2014;32:785-792.

14. Monroe AK, Brown TT, Cox C, et al. Physical activity and its association with insulin resistance in multicenter AIDS cohort study men. AIDS Res Hum Retroviruses 2015;31:1250-1256

15. Rehm KE, Konkle-Parker D. Physical activity levels and perceived benefits and barriers to physical activity in HIV-infected women living in the deep south of the United States. AIDS Care 2016;28:1205-1210.

16. Webel AR, Barkley J, Longenecker CT, Mittelsteadt A, Gripshover B, Salata RA. A crosssectional description of age and gender differences in exercise patterns in adults living with HIV. The Journal of the Association of Nurses in AIDS Care: JANAC 2015;26: 176-186.

17. Kitahata MM, Rodriguez B, Haubrich R, et al. Cohort profile: the centers for AIDS research network of integrated clinical systems. Int J Epidemiol 2008;37:948-955.

18. Crane HM, Lober W, Webster E, et al. Routine collection of patient-reported outcomes in an HIV clinic setting: the first 100 patients. Curr HIV Res 2007;5:109-118.

19. Fredericksen R, Crane PK, Tufano J, et al. Integrating a web-based, patientadministered assessment into primary care for HIV-infected adults. Journal of AIDS and HIV research 2012;4:47-55.

20. Ainsworth BE, Jacobs Jr DR, Leon AS. Validity and reliability of self-reported physical activity status: the lipid research clinics questionnaire. Med Sci Sports Exerc 1993;25: 92-98.

21. Crane HM, Heckbert SR, Drozd DR, et al. Lessons learned from the design and implementation of myocardial infarction adjudication tailored for HIV clinical cohorts. Am Epidemiol 2014;179:996-1005. 
22. Crane HM, Kadane JB, Crane PK, Kitahata MM. Diabetes case identification methods applied to electronic medical record systems: their use in HIV-infected patients. Curr HIV Res 2006;4:97-106.

23. Stengel B, Tarver-Carr ME, Powe NR, Eberhardt MS, Brancati FL. Lifestyle factors, obesity and the risk of chronic kidney disease. Epidemiology 2003;14:479-487.

24. Thune I, Furberg AS. Physical activity and cancer risk: dose-response and cancer, all sites and site-specific. Medicine and Science in sports And Exercise 2001;33:S530S550. (discussion S609-510).

25. Vincent L, Leedy D, Masri SC, Cheng RK. Cardiovascular disease and cancer: is there increasing overlap? Curr Oncol Rep 2019;21:47.

26. Maggiolo F, Roat E, Pinti M, et al. Mitochondrial changes during D-drug-containing once-daily therapy in HIV-positive treatment-naive patients. Antivir Ther 2010;15: 51-59.

27. Dalakas MC. Peripheral neuropathy and antiretroviral drugs. Journal of the Peripheral Nervous System: JPNS 2001;6:14-20.

28. Gelman A, Hill J, Yahiman M. Why we (usually) don't have to worry about multiple comparisons. J Research on Educational Effectiveness 2012;5:189-211.

29. U.S. Department of Health and Human Services CfDCaP, National Center for Chronic Disease Prevention and Health Promotion, Division of Nutrition, Physical Activity and Obesity, ed. Nutrition, physical activity, and obesity data, trends and maps web site, vol 2016. ; 2015.. Atlanta, GA.

30. Smith AD, Crippa A, Woodcock J, Brage S. Physical activity and incident type 2 diabetes mellitus: a systematic review and dose-response meta-analysis of prospective cohort studies. Diabetologia 2016;59(12):2527-2545

31. Yarasheski KE, Cade WT, Overton ET, et al. Exercise training augments the peripheral insulin-sensitizing effects of pioglitazone in HIV-infected adults with insulin resistance and central adiposity. Am J Physiol Endocrinol Metab 2011;300:E243-E251.

32. Han JH, Crane HM, Bellamy SL, et al. HIV infection and glycemic response to newly initiated diabetic medical therapy. Aids 2012;26:2087-2095.

33. Xu Y, Chen X, Wang K. Global prevalence of hypertension among people living with HIV: a systematic review and meta-analysis. J Am Soc Hypertens 2017;11:530-540.

34. Yu S, Yarnell JW, Sweetnam PM, Murray L, Caerphilly S. What level of physical activity protects against premature cardiovascular death? The Caerphilly study. Heart 2003;89:502-506
35. Hand GA, Lyerly GW, Jaggers JR, Dudgeon WD. Impact of aerobic and resistance exercise on the health of HIV-infected persons. American Journal of Lifestyle Medicine 2009;3:489-499.

36. Kelley GA, Kelley KS. Aerobic exercise and lipids and lipoproteins in men: a metaanalysis of randomized controlled trials. The Journal of Men's Health \& Gender: The Official Journal of The International Society for Men's Health \& Gender 2006;3:61-70.

37. Kelley GA, Kelley KS, Tran ZV. Aerobic exercise and lipids and lipoproteins in women: a meta-analysis of randomized controlled trials. J Womens Health 2004;13:11481164.

38. Willig AL, Kramer PA, Chacko BK, Darley-Usmar VM, Heath SL, Overton ET. Monocyte bioenergetic function is associated with body composition in virologically suppressed HIV-infected women. Redox Biol 2017;12:648-656.

39. Campbell PJ, Aurelius S, Blowes G, Harvey D. Decrease in CD4 lymphocyte counts with rest; implications for the monitoring of HIV infection. Int J STD AIDS 1997;8: 423-426.

40. Weng TP, Huang SC, Chuang YF, Wang JS. Effects of interval and continuous exercise training on CD4 lymphocyte apoptotic and autophagic responses to hypoxic stress in sedentary men. PloS One 2013;8, e80248.

41. Zanetti HR, Cruz LG, Lourenco CL, Neves Fde F, Silva-Vergara ML, Mendes EL. Nonlinear resistance training reduces inflammatory biomarkers in persons living with HIV: a randomized controlled trial. Eur J Sport Sci 2016;16:1232-1239.

42. O'Brien KK, Tynan AM, Nixon SA, Glazier RH. Effectiveness of aerobic exercise for adults living with HIV: systematic review and meta-analysis using the Cochrane Collaboration protocol. BMC Infect Dis 2016;16:182.

43. Saffer H, Dave D, Grossman M, Leung LA. Racial, ethnic, and gender differences in physical activity. Journal of Human Capital 2013;7:378-410.

44. Fredriksen-Goldsen KI, Cook-Daniels L, Kim HJ, et al. Physical and mental health of transgender older adults: an at-risk and underserved population. Gerontologist 2014:54:488-500.

45. Smalley KB, Warren JC, Barefoot KN. Differences in health risk behaviors across understudied LGBT subgroups. Health Psychol 2016;35:103-114.

46. Ahmad MH, Salleh R, Mohamad Nor NS, et al. Comparison between self-reported physical activity (IPAQ-SF) and pedometer among overweight and obese women in the MyBFF@home study. BMC Womens Health 2018;18:100. 